\title{
Direcionadores da adoção de estratégias de postponement: estudo multicaso em empresas da indústria de alimentos
}

\author{
Karine Araújo Ferreira ${ }^{\mathrm{a} *}$, Rosane Lucia Chicarelli Alcântara ${ }^{\mathrm{b}}$ \\ a*karine@deenp.ufop.br, UFOP, Brasil \\ brosane@dep.ufscar.br, UFSCar, Brasil
}

\begin{abstract}
Resumo
0 propósito deste artigo foi investigar a aplicação prática do postponement em empresas alimentícias brasileiras e identificar os direcionadores que motivam/facilitam a adoção do postponement nessas empresas. Seis estudos de caso foram realizados em empresas do segmento de fabricação de conservas de frutas e vegetais, como empresas processadoras de suco laranja e de derivados de tomate. Além de maior discussão sobre o tema e a verificação de sua aplicação em empresas alimentícias, este trabalho traz duas contribuições principais: a primeira é apresentar um quadro teórico com os principais direcionadores para a adoção do postponement identificados na literatura; a segunda é confrontar os direcionadores da pesquisa empírica e teórica, gerando um quadro teórico com os principais direcionadores adaptado para indústria de alimentos.
\end{abstract}

Palavras-chave

Postergação. Direcionadores. Gestão da cadeia de suprimentos.

\section{Introdução}

Um dos desafios das empresas modernas consiste em produzir exatamente o que os consumidores desejam, no local apropriado, a custo e no tempo desejados pelo consumidor (WIKNER; NAIM; RUDBERG, 2007). Para Van Hoek, Peelen e Comandeur (1999), uma maneira de enfrentar esse desafio consiste em obter benefícios pela economia de escala para determinados estágios de conformação de produtos, deixando a etapa de diferenciação para o ponto em que a demanda é conhecida. Envolve também permitir que produtos sejam estocados em armazéns centralizados até o momento em que o pedido é feito. Essa estratégia conhecida como postponement é definida por Van Hoek (2001) como estratégia da cadeia de suprimentos que consiste em adiar o máximo possivel qualquer movimentação e/ou configuração final de produtos. Assim, o produto não é deslocado até que a localização da demanda (ou ponto de consumo) seja conhecida, ao mesmo tempo em que sua configuração final (customização) só acontece quando as preferências do consumidor são conhecidas. lsto possibilita trazer o ponto no qual decisões sobre 0 que fazer são tomadas para mais perto do recebimento dos pedidos do consumidor, resultando em estoques e custos logísticos reduzidos (ASSUMPÇÃO; RIBEIRO, 2001; VAN HOEK, 2001; YANG; BURNS; BACKHOUSE, 2004a). Adicionalmente, a postergação de tarefas ao longo da cadeia de suprimentos constitui possível estratégia para o alcance da customização em massa (VAN HOEK; COMMANDEUR; VOS, 1998; VAN HOEK; PEELEN; COMMANDEUR, 1999; VAN HOEK; 2001; MACHADO; MORAES, 2008).

As primeiras experiências utilizando o postponement remontam à década de 1920 (COUNCIL..., 1995) e, na literatura acadêmica, o termo foi introduzido primeiramente por Alderson (1950) como uma forma de ordenamento de etapas de agregação de valor nos processos de manufatura e de marketing.

Nos últimos anos, o presente tema tem despertado interesse de acadêmicos e executivos em diversos paises. Trabalhos abordando o conceito postponement podem ser encontrados em diferentes áreas, como 
logística, produção, marketing e, mais recentemente, na cadeia de suprimentos, conforme revisão bibliográfica realizada por Yang, Burns e Backhouse (2004a). Apesar da atenção e da discussão crescente da aplicação dessa estratégia em setores como confecções, eletrônicos e automotivo, poucos estudos foram feitos sobre a viabilidade e o uso da teoria do postponement no setor alimentício, onde o conceito ainda é pouco discutido. Nesse setor foram encontrados poucos trabalhos internacionais abordando o tema: um em empresas alimentícias da Europa; outro em uma empresa de vinho europeia; e em uma revisão bibliográfica (VAN HOEK, 1997, 1999; VAN DONK, 2001). No Brasil, faltam pesquisas mais aprofundadas sobre o tema na indústria de alimentos. Existem estudos teóricos, como o trabalho de Assumpção e Ribeiro (2001) e o estudo desenvolvido por Sampaio (2003) que, no segmento alimentício, investiga o tema em uma doceria. Porém verifica-se a falta de publicações sobre resultados de pesquisas ou estudos empíricos aprofundados em empresas desse segmento.

Assim, este artigo tem o propósito de investigar a implementação da estratégia de postponement em empresas brasileiras de alimentos, buscando responder as seguintes questões:

- Quais tipos de postponement são adotados e como é a sua aplicação em empresas alimentícias?

- Quais os direcionadores da adoção dessa estratégia nas empresas da indústria de alimentos?

Como a implementação do postponement ainda se encontra pouco discutida, principalmente na indústria de alimentos, respostas a essas questões são relevantes. Como principal contribuição teórica, este artigo apresenta um quadro contendo os principais direcionadores para a adoção do postponement identificados na literatura, o qual pode e deve ser adequado para diferentes setores da indústria. Em uma perspectiva empírica, este artigo visa, além de investigar a implementação da estratégia de postponement em empresas brasileiras de alimentos, confrontar os direcionadores obtidos na teoria com os adotados na prática, gerando assim, um quadro com os principais direcionadores desta estratégia adaptado para empresas alimentícias.

Este trabalho também se justifica pela importância da indústria alimentícia na atividade econômica brasileira. Segundo a ABIA (ASSOCIAÇÃO..., 2011), o setor faturou $\mathrm{R} \$ 388,7$ bilhões e criou mais de 94 mil novos postos de trabalho em 2011, o que demonstra sua representatividade. Corrobora ainda o fato de ser esse setor um dos principais exportadores de produtos, como é o caso do suco de laranja concentrado e congelado, um dos segmentos foco deste estudo. 0 Brasil é atualmente o maior produtor e exportador mundial do suco de laranja concentrado.
Adicionalmente, ao se pesquisar o setor alimentício, pode-se constatar que existem significativas diferenças neste setor em comparação com outros setores da indústria. As diferenças existentes decorrem das características específicas do setor alimentício, destacadas por Van Donk (2001), Van Hoek (1999) e Twede, Clarke e Tai (2000) em três dimensões: a) características do mercado; b) características do produto; e c) características do processo. Em relação ao mercado alimentício, os mesmos autores destacam que esse é caracterizado por: incerteza da demanda; curto tempo de entrega; grande variedade de produtos; e alta concorrência. São características que distinguem os produtos das empresas processadoras de alimentos a qualidade e o preço variáveis, devido à sazonalidade e ao fato de os produtos agrícolas estarem sujeitos a flutuações e variações naturais do tempo que influenciam não somente a preferência dos consumidores, mas também afetam o rendimento no suprimento dos produtos. Adicionalmente, matériasprimas, produtos semiacabados e produtos finais em geral são pereciveis e de baixo valor agregado. Por fim, em relação às características do processo, Van Hoek (1999) e Twede, Clarke e Tai (2000) afirmam que, geralmente, empresas de alimentos apresentam processos de produção contínuos e não modulares, o que interpõe à aplicação do postponement, em alguns segmentos da indústria de alimentos, um desafio especial.

Este artigo está estruturado em seis seções, incluindo a introdução. Na seção 2, é apresentada uma síntese da literatura sobre postponement. 0 método de pesquisa adotado é discutido na seção 3. Os principais resultados dos estudos de caso são expostos na seção 4 e, na seção 5, eles são analisados e discutidos. Por fim, são apresentadas as considerações finais do trabalho (seção 6) e as referências bibliográficas.

\section{Postponement}

Nesta seção é apresentada uma revisão bibliográfica sobre postponement, destacando as definições, os tipos e os principais direcionadores da adoção dessa estratégia. Esses direcionadores identificados na literatura foram analisados, ordenados e reagrupados em um quadro teórico elaborado nesta pesquisa e que é apresentado ao final dessa seção.

\subsection{Definição e tipos de postponement}

0 conceito postponement foi introduzido na literatura acadêmica por Alderson (1950) como uma maneira de mudar a forma, identidade ou lugar 
de produtos em um momento tão tardio quanto possível dentro dos processos de manufatura e distribuição física. Assim, postergar a movimentação do produto foi denominado postponement de tempo (time postponement), enquanto a postergação na diferenciação do produto foi denominada postponement de forma (form postponement).

Em 1965, Bucklin agregou mais detalhes ao trabalho de Alderson, estudou limites de aplicação da estratégia e criou o princípio da especulação (principle of speculation), conceito oposto ao postponement. Esse princípio é o inverso do postponement: consiste em finalizar as operações o mais cedo possível no processo de manufatura (BUCKLIN, 1965).

Depois de 1965, poucos trabalhos abordaram o assunto. 0 tema foi retomado por Zinn e Bowersox (1988), que propuseram que o postponement poderia ser separado em cinco diferentes tipos, quatro relacionados com alterações de forma do produto (etiquetagem, embalagem, montagem e manufatura) e o quinto relacionado ao tempo (centralização dos estoques).

Para Bowersox e Closs (1996) existem dois tipos de postponement: o postponement de manufatura (ou forma) e o postponement logístico (de tempo). 0 postponement de manufatura consiste em levar a fabricação de um produto base ou padrão a quantidades suficientes que se realize economia de escala, enquanto as características de finalização são adiadas até os pedidos dos consumidores serem recebidos. Já o postponement logístico consiste em manter toda linha de produtos já acabados em um estoque centralizado. Igualmente, o deslocamento de estoques é adiado até os pedidos dos clientes serem recebidos. Quando a demanda ocorre, os produtos são transportados diretamente ao varejo ou ao consumidor.

Pagh e Cooper (1998) combinaram quatro estratégias de postponement para uma cadeia de suprimentos em uma matriz 2x2 (Quadro 1). Nessa matriz, postponement de forma é chamado postponement de manufatura (manufacturing postponement) e postponement de tempo é chamado postponement logístico (logistic postponement). As quatro estratégias são então desenvolvidas pela combinação do postponement de manufatura e logística. As duas primeiras são representadas tanto pelo postponement de manufatura como pelo postponement logístico. As outras duas são formadas pela combinação de ambas, denominada de estratégia de postponement total, e pela ausência de ambas, denominada de estratégia de especulação completa.

Waller, Dabholkar e Gentry (2000), Cardoso (2002), Yang, Burns e Backhouse (2004b), Garcia-Dastugue e Lambert (2007) e outros autores de diferentes correntes literárias também propõem outras classificações de postponement, ampliando o leque de possibilidades.

Em síntese, pôde-se verificar que existem várias tipologias de postponement, envolvendo múltiplos elementos e diferentes aspectos. Isso é natural, uma vez que existem várias combinações possíveis de adiamento espaço-temporal e que trabalhos sobre 0 tema podem ser encontrados em diferentes correntes literárias.

Nesta pesquisa, adotou-se a classificação original proposta por Alderson (1950), ou seja, buscou-se investigar se as empresas analisadas adotavam o postponement de forma e/ou tempo. Esta classificação foi adotada porque embora diferentes denominações e classificações de postponement tenham sido elaboradas pelos autores ao longo dos anos, grande parte delas tem o mesmo significado, sendo apenas um novo rótulo para tipos de postponement propostos por Alderson (1950). Por exemplo, o postponement de manufatura descrito por Bowersox e Closs (1996) e Pagh e Cooper (1998) ou, ainda, o postponement de produção proposto por Yang, Burns e Backhouse (2004b) são apenas um novo rótulo para o postponement de forma, sendo o significado do conceito o mesmo, que é a postergação na configuração dos produtos até que a demanda seja conhecida. Assim, optou-se por adotar a classificação original do conceito apresentado por Alderson (1950).

Além dos tipos de postponement, foram identificadas nesta pesquisa em quais atividades o postponement acontece: operações e fabricação, manufatura final, embalagem, etiquetagem, logística (distribuição física); bem como em qual estágio da cadeia de suprimentos o postponement acontece.

Quadro 1. Matriz postponement/especulação e estratégias genéricas da cadeia de suprimentos.

\begin{tabular}{|l|l|l|l|}
\hline \multicolumn{2}{|c|}{} & \multicolumn{2}{|c|}{ Logística } \\
\hline \multirow{2}{*}{ Manufatura } & $\begin{array}{l}\text { Especulação } \\
\text { Estoques descentralizados }\end{array}$ & $\begin{array}{l}\text { Postponement } \\
\text { Estoques centralizados e distribuição direta }\end{array}$ \\
\cline { 2 - 4 } & $\begin{array}{l}\text { Especulação } \\
\text { Fabricação para estocagem }\end{array}$ & Estratégia de especulação total & Estratégia de postponement de logística \\
\hline Fabricação por pedidos & Estratégia de postponement de manufatura & Estratégia de postponement total \\
\hline
\end{tabular}

Fonte: Adaptado de Pagh e Cooper (1998, p. 15). 


\subsection{Direcionadores da aplicação de postponement}

A decisão de quando (em que situações) aplicar o postponement está diretamente associada à razão pela qual a empresa deve aplicá-lo. Vários autores, como Ballou (2001) e Van Hoek (1999) frisam a importância da organização definir, antes de tudo, seus objetivos. A adoção do postponement deve ser adequada para o alcance desses objetivos e é significativamente influenciada e facilitada por características operacionais e logísticas da empresa, bem como pelo ambiente de negócios no qual ela se insere.

Na literatura sobre postponement, vários autores têm destacado diferentes fatores que favorecem/ facilitam a adoção do postponement, ou seja, direcionadores de sua adoção. Zinn e Bowersox (1988), por exemplo, usando a metodologia de simulação puderam quantificar os trade-offs que influenciam a decisão da empresa de aplicar o postponement e puderam observar que fatores direcionam as decisões de postergação. Eles concluíram que dois drivers são comuns para todos os tipos de postergação: incerteza da demanda e valor do produto. Outros direcionadores identificados pelos mesmos autores foram: economia de transporte; elevado número de marcas e versões do produto; variações de peso e tamanho do produto; e alto percentual de materiais onipresentes.

Van Hoek (1997) listou três importantes precondições para a implementação do postponement ser conduzida com sucesso: possibilidade de o processo de manufatura ser dividido em duas ou mais etapas distintas (desacoplamento de processos); modularidade; e o escopo do processo final de montagem. Já Pagh e Cooper (1998) desenvolveram o conceito de análise do perfil (Profile Analysis), em que os principais direcionadores considerados significativos para implementação do postponement foram: estágio do ciclo de vida do produto; volume; estratégia de custo/serviço; tipo de produto; variedade; perfil de valor; densidade monetária; tempo de entrega: frequência de entrega; nível de instabilidade da demanda; economia de escala; e complexidade da personalização.

Em outro artigo, Van Hoek, Comandeur e Vos (1998) identificaram um conjunto de direcionadores que favorecem a implementação da estratégia de postponement de manufatura dentro das características do processo e da tecnologia, do produto e do mercado, como mostra o Quadro 2.

Em sua análise, Johnson e Anderson (2000) mostraram que o postponement é uma estratégia adequada para produtos com ciclos de vida curtos. Já Twede, Clarke e Tai (2000) encontraram diversos direcionadores que indicam que uma estratégia de postponement de embalagem pode ser apropriada. São eles: (a) produtos modulares que podem ser customizados para mercados locais; (b) produtos que ganham volume, peso ou valor com a embalagem; (c) demanda imprevisível; (d) um grande número de variações baseadas no mercado para formulação única; (e) situações em que economia da escala para produção e logística pode ser utilizada. Chiou, Wu e Hsu (2002) identificaram quatro principais características do produto/demanda que podem ser destacadas como direcionadoras da estratégia de postponement de forma. Esses facilitadores são: demanda para customização; modularidade; valor do produto; e ciclo de vida do produto.

Cardoso (2002) relacionou direcionadores e categorias de direcionadores (dimensões), elaborando pressupostos sobre a aplicação do postponement.

Quadro 2. Direcionadores da implementação do postponement de manufatura.

\begin{tabular}{|c|c|}
\hline Direcionadores & Motivo \\
\hline $\begin{array}{l}\text { Caracteristicas do processo e tecnologia } \\
\text { Processo modular, permitindo o seu desacoplamento em } \\
\text { atividades primárias e secundárias } \\
\text { Pouca complexidade na operação de personalização } \\
\text { Projeto modular } \\
\text { Fontes de múltiplas locações }\end{array}$ & $\begin{array}{l}\text { Precondição } \\
\text { Reduz os problemas decorrentes da perda economia de escala } \\
\text { Rápida personalização a baixo custo } \\
\text { Embarque direto de módulos }\end{array}$ \\
\hline $\begin{array}{l}\text { Caracteristicas do produto } \\
\text { Módulos intercambiáveis } \\
\text { Formulação específica do produto } \\
\text { Periféricos específicos } \\
\text { Produto de alto valor monetário } \\
\text { Aumento de peso ou volume } \\
\end{array}$ & $\begin{array}{l}\text { Reduz níveis de estoques e reduz riscos de obsolescência } \\
\text { Melhora a personalização } \\
\text { Melhora a personalização } \\
\text { Reduz os custos de armazenagem } \\
\text { Reduz os custos de transporte }\end{array}$ \\
\hline $\begin{array}{l}\text { Caracteristicas do mercado } \\
\text { Ciclo de vida curto } \\
\text { Alta oscilação da demanda } \\
\text { Necessidade de lead time e confiável } \\
\text { Competição em preço } \\
\text { Mercado segmentado }\end{array}$ & $\begin{array}{l}\text { Reduz o risco de obsolescência dos inventários } \\
\text { Reduz o nível de estoques } \\
\text { Melhoria no nível de serviço } \\
\text { Reduz os custos } \\
\text { Melhora o posicionamento do produto no mercado e segmentação }\end{array}$ \\
\hline
\end{tabular}

Fonte: Van Hoek, Comandeur e Vos (1998, p. 37-38). 
Para a mesma autora, são determinantes na aplicação do postponement: longo lead time de produção; pouca quantidade de produtos; nível do serviço ao cliente; grande variedade de produtos; incerteza de demanda; alto valor do produto; alta correlação de vendas entre produtos da mesma linha; magnitude; nível de customização de produto; incerteza de lead time; incerteza no tempo de entrega; curto ciclo de vida do produto; falta de infraestrutura de transporte e comunicação; aplicação de políticas governamentais; elevado custo de manutenção de estoques; elevado custo de armazenagem; elevado custo de transporte/ distribuição; elevado custo de processamento de pedidos; baixo custo de vendas perdidas; elevado custo de produção. Além desses direcionadores, Cardoso (2002) destacou, ainda: características do produto; mercado/demanda; tecnologia/processo produtivo; e características do sistema logístico como principais dimensões determinantes para a aplicação do postponement.

Sampaio (2003) investigou a aplicação do postponement em cinco empresas (duas automobilísticas, uma de tintas, uma de computadores e uma doceria) e destacou os direcionadores que favoreceriam a aplicação do postponement no Brasil. Esses foram agrupados em dimensões relacionadas a: produto; processo; mercado; cadeia de suprimentos; liderança; e tecnologia, no Quadro 3.

De acordo com Matthews e Syed (2004), as companhias que desejam empregar estratégias de postponement de forma bem sucedida devem ter certas características. Primeiramente, as empresas devem adotar projetos de produtos padronizados, reengenharia de processos de negócio padronizados e colaboração interfuncional para suportar tempos de resposta mais rápidos e facilitar a visibilidade na cadeia de suprimentos. Além disso, o postponement é mais eficaz quando as companhias têm implementado processos colaborativos com os parceiros comerciais. Também é essencial para o postponement a habilidade de a companhia medir e manter níveis de desempenho do serviço e da entrega competitivos. Em termos de características do negócio, os melhores candidatos à aplicação do postponement produzem uma variedade significativa de produtos com ciclos de vida curtos e têm uma cadeia de suprimentos local que pode suportar customização em massa. Isso requer também treinamento para aplicação do postponement, um plano de gestão da mudança na empresa e uso de tecnologias de informação e gestão empresarial.

Para Yang, Burns e Backhouse (2004a), a identificação de características do mercado, do produto e da produção é essencial para a aplicação bem sucedida do postponement. Eles destacam os seguintes direcionadores como relevantes para implementação da estratégia: elevada flutuação das vendas e ciclo de vida dos produtos curto (características do marketing); elevado grau de customização, comonalidade ou modularidade (características do produto); desacoplamento do processo do produto (característica da produção).

Em um artigo posterior, Yang, Burns e Backhouse (2005) afirmam que muitas razões são apontadas para a crescente importância das estratégias de postponement, dentre elas:

- Ciclos de vida do produto mais curtos;

- Propagação do produto;

- Desenvolvimento tecnológico sempre contínuo;

- Consumidores mais sofisticados;

- Sucesso guiado não somente pelo custo ou qualidade mas pela velocidade;

- Maior interesse dos pesquisadores e praticantes em customização em massa e agilidade;

- Expansão de sistemas de informação e comunicação e surgimento do comércio eletrônico;

- Arquitetura do produto e dos processos de produção;

- Padronização e projeto modular; e

- Foco na melhoria da cadeia de suprimentos como um todo.

Mais recentemente, Yang, Yang e Wijngaard (2007) discutem a importância do planejamento de capacidade e da estrutura interorganizacional para

Quadro 3. Direcionadores que favorecem a aplicação do postponement no Brasil.

\begin{tabular}{|l|l|}
\hline \multicolumn{1}{|c|}{ Dimensão } & \multicolumn{1}{c|}{ Aspectos } \\
\hline Produto & Modularidade; Formulação específica do produto; Complexidade e customização final; Densidade monetária \\
\hline Processo & $\begin{array}{l}\text { Processo modular; Processos produtivos sobrecarregados; Processos de manufatura flexíveis; Pulmão estratégico; } \\
\text { Economia de escala }\end{array}$ \\
\hline Mercado & $\begin{array}{l}\text { Variação da demanda; Volume; Estágio do ciclo de vida; Ciclo de vida; Tempo de entrega; Frequência de } \\
\text { entrega; Adoção }\end{array}$ \\
\hline Cadeia de suprimentos & $\begin{array}{l}\text { Relacionamento colaborativo; Resposta rápida dos fornecedores; Proximidade com os fornecedores; } \\
\text { Sequenciamento de peças; Legislação; Treinamento; Sistema pós-venda }\end{array}$ \\
\hline Liderança & Estratégia da organização; Comprometimento \\
\hline Tecnologia & E-commerce; Sistema de pagamento; Fornecedores de equipamentos \\
\hline
\end{tabular}

Fonte: adaptado de Sampaio (2003). 
aplicação da estratégia de postponement. Para eles, a configuração dos relacionamentos na cadeia de suprimentos é essencial à implantação do conceito.

Baseado nessa revisão é possível observar que diferentes direcionadores para a aplicação do postponement são encontrados na literatura. $\mathrm{Na}$ próxima seção, esses direcionadores são analisados, classificados e agrupados em diferentes dimensões.

\subsection{Construção do quadro conceitual}

A seguir será apresentado o quadro conceitual desenvolvido para esta pesquisa, que tem como base os direcionadores analisados na fundamentação teórica desenvolvida na seção 2.2. Este quadro é composto por sete dimensões (identificadas na seção 2.2) nas quais os direcionadores foram agrupados. São elas: mercado; produto; processo; logística/ manufatura; cadeia de suprimentos; liderança; e tecnologia. Uma vez identificadas essas dimensões, todos os direcionadores (fatores que favorecem e/ou facilitam a adoção do postponement) identificados na revisão da literatura foram analisados, ordenados, reagrupados e relacionados à dimensão que mais se adequavam, possibilitando assim a geração do quadro teórico apresentado nesta pesquisa (Quadro 4). Esses direcionadores foram posteriormente comparados aos encontrados nas pesquisas de campo, permitindo assim a elaboração de outro quadro contendo os direcionadores da adoção do postponement adaptado para empresas alimentícias, apresentado na seção 4.

\section{Método de pesquisa}

A pesquisa realizada neste trabalho é de abordagem qualitativa e o método adotado foi o estudo de caso. Segundo Yin (2001, p. 32),

[...] um estudo de caso é uma investigação empírica que estuda um fenômeno contemporâneo dentro do contexto da vida real, especialmente quando os limites entre fenômeno e contexto não estão claramente definidos [...].

0 foco desta pesquisa é direcionado para a situação presente, ou seja, em que amplitude as empresas alimentícias têm utilizado o postponement e quais fatores caracterizam e possibilitam a aplicação desse conceito.

Os estudos de caso desenvolvidos nesta pesquisa são de natureza exploratória, pois apesar de estudos acadêmicos sobre o tema serem feitos há mais de 60 anos, o processo de adoção da estratégia de postponement no ambiente de negócio brasileiro e principalmente nas empresas de alimentos é ainda pouco explorado. Além disso, esta pesquisa foi desenvolvida em múltiplos casos, para aumentar o grau de validade externa e também reduzir qualquer viés do pesquisador.

Foi definida unidade de análise para o estudo de caso a empresa processadora de alimentos (setor industrial) que adotasse um ou mais tipos de postponement identificados na literatura. Conforme mencionado, esse setor foi escolhido devido a falta de pesquisas acadêmicas que abordem a aplicação do postponement nele. Foram então selecionadas para esta pesquisa seis empresas processadoras de alimentos, sendo três da subclasse fabricação de sucos de frutas e outras três da subclasse fabricação de conservas de frutas. Essas empresas foram escolhidas como objeto de estudo por atenderem aos seguintes requisitos da pesquisa: a) serem empresas processadoras de alimentos; b) adotarem algum tipo de postponement; c) estarem disponíveis para a pesquisa; d) possuírem características comuns para efeito de comparação; e) serem representativas dentro do segmento. Adicionalmente, elas têm a maioria de seus produtos inseridos em uma mesma classe ou segmento denominado fabricação de conservas de frutas, legumes e outros vegetais, de acordo com a Classificação Nacional de Atividades Econômicas (COMISSÃO..., 2008).

Esta pesquisa foi conduzida por entrevistas semiestruturadas realizadas em cada empresa com pelo menos dois gerentes responsáveis pelas áreas da produção ou da logística. Os estudos de caso foram realizados entre agosto de 2008 e agosto de 2009, envolvendo sempre a entrevista com duração média de 1,5 a 2 horas. Também foram utilizadas nesta pesquisa fontes de dados adicionais como relatórios contendo informações sobre aplicação do postponement e observação direta, que foi realizada de maneira informal nas visitas realizadas às empresas para coleta de dados.

Baseado no quadro teórico contendo os direcionadores para aplicação do postponement identificados da literatura (Quadro 4), foi solicitado aos entrevistados que indicassem se algum dos direcionadores identificados na teoria se aplicava na sua empresa e se haviam outros. Adicionalmente, foi solicitado que indicassem a contribuição de cada direcionador indicado. Para tanto, a contribuição desses direcionadores para a adoção do postponement foi numerada e classificada como 4 - muito alta; 3 - alta; 2 - média; 1 - baixa; 0 - não se aplica/ ou não contribuiu/ou não foi citado pela empresa.

0 material coletado (nas entrevistas semiestruturadas, relatórios e observação direta) sob escopo das teorias permitiu elaborar um esquema de interpretação dos fenômenos estudados. Esse esquema se apoiou em três aspectos fundamentais: 
Quadro 4. Direcionadores da adoção do postponement agrupados por dimensão.

\begin{tabular}{|c|c|}
\hline Dimensão & Fatores \\
\hline Mercado & $\begin{array}{l}\text { Incerteza da demanda (ZINN; BOWERSOX, 1988; PAGH; COOPER, 1998; JOHNSON; ANDERSON, 2000; CARDOSO, } \\
\text { 2002; SAMPA1O, 2003; YANG; BURNS; BACKHOUSE, 2004a) } \\
\text { Variação da demanda (VAN HOEK; COMANDEUR; VOS, 1998; SAMPA10, 2003) } \\
\text { Demanda para customização (CHIOU; WU; HSU, 2002; CARDOSO, 2002) } \\
\text { Segmento de mercado (VAN HOEK; COMANDEUR; VOS, 1998) } \\
\text { Consumidores exigentes (YANG et al., 2005) }\end{array}$ \\
\hline Produto & $\begin{array}{l}\text { Tipo do produto (PAGH; COOPER, 1998) } \\
\text { Preço (ZINN; BOWERSOX, 1988; VAN HOEK; COMANDEUR; VOS, 1998; PAGH; COOPER, 1998; JOHNSON; ANDERSON, } \\
\text { 2000; TWEDE; CLARKE; TAl 2000; CHIOU; WU; HSU, 2002; CARDOSO, 2002) } \\
\text { Marcas e versões do produto (ZINN; BOWERSOX, 1988; CARDOSO, 2002) } \\
\text { Variação de tamanho (ZINN; BOWERSOX, 1988; VAN HOEK; COMANDEUR; VOS, 1998; CARDOSO, 2002; SAMPA10, } \\
\text { 2003) } \\
\text { Variação de peso (ZINN; BOWERSOX, 1988; VAN HOEK; COMANDEUR; VOS, 1998; CARDOSO, 2002; SAMPA10, 2003) } \\
\text { Formulação específica do produto (VAN HOEK; COMANDEUR; VOS, 1998; SAMPA10, 2003) } \\
\text { Periféricos específicos (VAN HOEK; COMANDEUR; VOS, 1998) } \\
\text { Modularidade/Comonalidade (VAN HOEK, 1997; VAN HOEK; COMANDEUR; VOS, 1998; TWEDE; CLARKE; TAl 2000; } \\
\text { CH1OU; WU; HSU, 2002; SAMPAlO, 2003; YANG et al., 2005) } \\
\text { Padronização (YANG et al., 2005) } \\
\text { Modelos intercambiáveis (VAN HOEK; COMANDEUR; VOS, 1998) } \\
\text { Sequenciamento de peças (SAMPAIO, 2003) } \\
\text { Complexidade e customização em massa (PAGH; COOPER, 1998; CARDOSO, 2002; SAMPA10, 2003; YANG et al., 2005) } \\
\text { Ciclo de vida (VAN HOEK; COMANDEUR; VOS, 1998; TWEDE; CLARKE; TAl 2000; SAMPAIO, 2003; YANG; BURNS; } \\
\text { BACKHOUSE, 2004a, b) } \\
\text { Estágio do ciclo de vida (PAGH; COOPER, 1998; SAMPAIO, 2003) }\end{array}$ \\
\hline Processo & $\begin{array}{l}\text { Processo modular (ZINN; BOWERSOX, 1988) } \\
\text { Processos de manufatura flexíveis (SAMPA10, 2003) } \\
\text { Complexidade tecnológica do processo (VAN HOEK, 1997; VAN HOEK; COMANDEUR; VOS, 1998) } \\
\text { Reengenharia do processo de negócio (SAMPAIO, 2003; MATTHEWS; SYED, 2004) } \\
\text { Planejamento de capacidade (YANG; YANG; WIJNGAARD, 2007) } \\
\text { Economia de escala (PAGH; COOPER, 1998; TWEDE; CLARKE; TAl 2000; SAMPA1O, 2003) } \\
\text { Custo da produção (CARDOSO, 2002) } \\
\text { Lead time (VAN HOEK; COMANDEUR; VOS, 1998; CHIOU; WU; HSU, 2002; CARDOSO, 2002) } \\
\text { Desacoplamento de processos (VAN HOEK; COMANDEUR; VOS, 1998; YANG; BURNS; BACKHOUSE, 2004a) }\end{array}$ \\
\hline Logística & $\begin{array}{l}\text { Custo de estoque (CARDOSO, 2002) } \\
\text { Custo de armazenagem (CARDOSO, 2002) } \\
\text { Custo de transporte/distribuição (CARDOSO, 2002) } \\
\text { Custo de vendas perdidas (CARDOSO, 2002) } \\
\text { Infraestrutura de transporte (CARDOSO, 2002 } \\
\text { Serviço ao cliente (CARDOSO, 2002; SAMPA10, 2003) } \\
\text { Tempo de entrega (PAGH; COOPER, 1998) } \\
\text { Frequência de entrega (PAGH; COOPER, 1998; SAMPA10, 2003) } \\
\text { lncerteza de tempo de ressuprimento (CARDOSO, 2002) }\end{array}$ \\
\hline $\begin{array}{l}\text { Cadeia de } \\
\text { Suprimentos }\end{array}$ & $\begin{array}{l}\text { Colaboração interfuncional (SAMPA10, 2003; MATTHEWS; SYED, 2004) } \\
\text { Colaboração com clientes/fornecedores (MATTHEWS; SYED, 2004) } \\
\text { Resposta rápida dos fornecedores (SAMPA10, 2003) } \\
\text { Políticas governamentais (CARDOSO, 2002; SAMPA10, 2003) } \\
\text { Treinamento e gestão da mudança (SAMPA10, 2003; MATTHEWS; SYED, 2004) } \\
\text { Medição de desempenho (MATTHEWS; SYED, 2004) }\end{array}$ \\
\hline Liderança & $\begin{array}{l}\text { Estratégia da organização (PAGH; COOPER, 1998; SAMPA10, 2003) } \\
\text { Comprometimento (SAMPA10, 2003) }\end{array}$ \\
\hline Tecnologia & $\begin{array}{l}\text { E-commerce (SAMPA10, 2003) } \\
\text { Infraestrutura de tecnologia da informação (MATTHEWS; SYED, 2004; YANG et al., 2005) }\end{array}$ \\
\hline
\end{tabular}

a) Na análise de cada caso individualmente; b) $\mathrm{Na}$ comparação entre os casos; c) Na comparação entre teoria e prática. Para sintetizar os resultados, nesta pesquisa serão apresentados somente a comparação dos casos (seção 4), bem como a comparação entre teoria e prática, destacada na seção 5 .

\section{Apresentação dos resultados}

A seguir são apresentados os resultados dos estudos de caso realizados em seis empresas do segmento fabricação de conservas de frutas. Por questão de sigilo, as três empresas processadoras de suco de laranja foram denominadas S1, S2 e S3 e as empresas produtoras de derivados de tomate, $\mathrm{T} 1, \mathrm{~T} 2$ e T3. Todas as empresas estão sediadas no interior do estado de São Paulo, com exceção da empresa T3, instalada no interior de Goiás.

Além dos derivados de tomate, conforme proporção destacada na Tabela 1, outros produtos integram a linha principal das empresas T2 e T3. Porém, como essas empresas têm em comum a produção 
Tabela 1. Perfil das empresas produtoras de suco de laranja e das empresas produtoras de derivados de tomate.

\begin{tabular}{|c|c|c|c|c|c|c|}
\hline Características & Empresa S1 & Empresa S2 & Empresa S3 & Empresa T1 & Empresa T2 & Empresa T3 \\
\hline $\begin{array}{l}\text { Principais } \\
\text { produtos }\end{array}$ & $\begin{array}{c}\text { Suco de laranja } \\
\text { concentrado } \\
\text { e congelado } \\
\text { (SLCC - 45\%); Suco } \\
\text { integral }(55 \%)\end{array}$ & SLCC (100\%) & $\begin{array}{l}\text { SLCC (95\%); Suco } \\
\text { integral (5\%) }\end{array}$ & $\begin{array}{l}\text { Derivados de } \\
\text { tomate }(70 \%)\end{array}$ & $\begin{array}{l}\text { Polpa e derivados } \\
\text { de tomate }(40 \%) \\
\text { e de goiaba }(40 \%)\end{array}$ & $\begin{array}{l}\text { Derivados de } \\
\text { tomate }(30 \%) \\
\text { e vegetais em } \\
\text { conserva }(40 \%)\end{array}$ \\
\hline Fundação & 1963 & 1991 & 1988 & 1955 & 1990 & 1955 \\
\hline $\begin{array}{l}\text { Unidades no } \\
\text { Brasil }\end{array}$ & 4 & 3 & 3 & 1 & 3 & 1 \\
\hline $\begin{array}{l}\text { Unidades no } \\
\text { exterior }\end{array}$ & 1 & 0 & 2 & 0 & 0 & 0 \\
\hline $\begin{array}{l}\text { Número de } \\
\text { funcionários }\end{array}$ & 1.700 & $\begin{array}{c}1.800 \text { na entressafra. } \\
3.000 \text { na safra }\end{array}$ & $\begin{array}{c}400 \text { na entressafra, } \\
3.000 \text { na safra }\end{array}$ & $\begin{array}{l}350 \text { funcionários } \\
\text { diretos }\end{array}$ & $\begin{array}{l}960 \text { funcionários } \\
\text { diretos }\end{array}$ & $\begin{array}{c}1.300 \text { funcionários } \\
\text { diretos }\end{array}$ \\
\hline $\begin{array}{l}\text { Áreas de } \\
\text { atuação }\end{array}$ & 98\% exportação & 99\% exportação & 95\% exportação & $\begin{array}{l}\text { Todo Brasil e } \\
\text { exportação }\end{array}$ & $\begin{array}{c}\text { Todo Brasil e } \\
\text { exportação (12\%) }\end{array}$ & $\begin{array}{c}\text { Todo Brasil e } \\
\text { exportação (8\%) }\end{array}$ \\
\hline $\begin{array}{l}\text { Parcela de } \\
\text { mercado }\end{array}$ & \multicolumn{3}{|c|}{$\begin{array}{c}\text { Entre as quatro maiores processadoras de } \\
\text { suco de laranja do Brasil }\end{array}$} & \multicolumn{3}{|c|}{$\begin{array}{c}\text { Entre as seis maiores processadoras de } \\
\text { derivados de tomate }\end{array}$} \\
\hline
\end{tabular}

de derivados de tomate, serão aqui agrupadas e denominadas empresas produtoras de derivados do tomate. As principais características dessas empresas foram sintetizadas e são apresentadas na Tabela 1.

\subsection{Aplicação do postponement em empresas alimentícias}

Durante as entrevistas foram solicitadas informações sobre as diferentes estratégias adotadas pelas empresas para responder às demandas de mercado, as quais podem variar de uma estratégia de especulação até um dos diferentes formatos de postponement identificados na literatura, assim como pode variar a forma de sua aplicação. Baseado na pesquisa de campo foi possível verificar que os tipos de postponement de tempo e forma são encontrados nas empresas de sucos e produtoras de derivados de tomate investigadas.

Com relação ao postponement de forma, ocorre nas empresas estudadas principalmente a partir das atividades de manufatura final. No caso das empresas processadoras de suco de laranja, as etapas de extração do suco da laranja são realizadas pelas empresas processadoras (empresas S1, S2 e S3) para estoque em formulação praticamente única para os dois principais tipos de suco produzidos: suco de laranja concentrado e congelado; e suco de laranja integral (pronto para beber). 0 suco é então transportado até o país do cliente, destino em que é customizado (geralmente por uma empresa cliente, responsável pelo processamento e distribuição final do suco). Assim, algumas atividades de manufatura final como mistura de diferentes tipos de sucos (também conhecida como processo de blendagem), diluição, adição de componentes responsáveis pelo sabor e aroma do suco, bem como adição de embalagem e rótulo são postergadas para empresas engarrafadoras, geralmente localizadas no exterior, sendo realizadas somente no momento em que a demanda é mais conhecida. Já para as empresas de derivados de tomate, o tomate é pré-processado (transformado em polpa) e permanece armazenado no estado semiacabado até a demanda ser conhecida. Baseado nas especificações de receita e demanda por determinado tipo de produto final (purê, extrato, molho, entre outros), essa polpa é então transformada no produto final (derivados do tomate). Esse mesmo processo de postergação ocorre para os derivados da goiaba (doce de goiaba, goiabada cascão, goiabada em calda, entre outros) na empresa T2.

Baseado nesses resultados foi possível verificar que embora na literatura Van Hoek (1999) afirme que o postponement de forma ocorra prioritariamente nas atividades de rotulagem e embalagem nas empresas alimentícias, foi possível verificar na pesquisa de campo que esses não são os únicos tipos de postponement que ocorrem neste setor. Nas empresas alimentícias investigadas nesta pesquisa, o postponement de forma na atividade de manufatura final também foi identificado como um dos mais aplicados. Acredita-se que essa diferença esteja relacionada aos segmentos investigados dentro da indústria de alimentos e à especificidade de cada um deles. Embora Van Hoek (1999) tenha investigado diferentes empresas no setor alimentício, tais como laticínios, indústrias de biscoitos, de barras de cereais e de alimentos congelados pré-preparados, as empresas do segmento de conservas de frutas investigadas nesta pesquisa não foram objeto de estudo do mesmo autor.

Embora o postponement de forma tenha se verificado principalmente a partir da atividade de manufatura final nas empresas investigadas, foi possível também identificar esse tipo de postponement 
ocorrendo a partir de diferentes atividades para diferentes produtos da organização, em menor proporção. Como exemplo, a empresa S1, que implementou o postponement a partir da atividade de manufatura final para o SLCC e a partir da atividade de embalagem para o suco integral, que é o suco natural, pronto para beber. Outro exemplo é a empresa T3, que adota o postponement de forma na atividade de manufatura final para o tomate e, para vegetais em conserva, no caso, o milho, na atividade de rotulagem.

É importante destacar que embora o postponement de forma seja adotado nas seis empresas investigadas, ele ocorre em diferentes atividades e locais na cadeia de suprimentos de cada uma delas. No caso das empresas de suco de laranja, as atividades de manufatura final como, por exemplo: diluição, adição de componentes de sabor e aroma, além das atividades de envase, adição de embalagem e rótulo só ocorrem à jusante da cadeia de suprimentos, sendo realizadas pelas empresas engarrafadoras sediadas no exterior. Para as empresas de derivados de tomate, as atividades geralmente são postergadas nas próprias unidades fabris, ou seja, internamente nas empresas T1, T2 e T3, atrasando o momento em que as diferenciações ocorrem.

Em relação ao postponement de tempo, percebe-se que este ocorre para todos os produtos das empresas investigadas, não apenas para o suco de laranja, derivados de tomate e de goiaba. Assim, os diferentes tipos de produtos são mantidos em armazéns localizados nas unidades produtoras das empresas investigadas e são deslocados para seu destino (empresas engarrafadoras, no caso das processadoras de suco de laranja, e supermercados e demais canais de distribuição, no caso das produtoras de derivados do tomate e de goiaba) somente após o recebimento do pedido do cliente.

Em sintese, pode-se dizer que foi possível verificar que os postponements de tempo e forma são encontrados nas empresas de sucos e produtoras de derivados de tomate investigadas, sendo que o postponement de forma ocorre principalmente a partir das atividades de manufatura final nas empresas estudadas.

\subsection{Direcionadores do postponement em empresas alimentícias}

Buscando responder a segunda questão de investigação desta pesquisa, a Tabela 2 apresenta os fatores considerados principais direcionadores para a aplicação do postponement nas empresas alimentícias investigadas, agrupados nas dimensões mercado; produto; processo; logística; gestão da cadeia de suprimentos; liderança; e tecnologia. Conforme já mencionado, a contribuição desses fatores à adoção do postponement foi numerada e classificada como: 4 - muito alta; 3 - alta; 2 - média; 1 - baixa; 0 - não se aplica/ou não contribuiu/ou não foi citado pela empresa.

Cabe ressaltar que as medidas destacadas em cinza são medidas acrescentadas pelos entrevistados durante a realização dos estudos de caso e que não foram identificadas previamente na literatura investigada. Já as medidas não destacadas em cinza são as medidas já identificadas previamente na revisão bibliográfica e que também foram verificadas na pesquisa de campo.

Conforme pode ser visualizado na Tabela 2 , a sazonalidade foi o único direcionador citado por todas as empresas investigadas como tendo contribuição muito alta para adoção do postponement. A sazonalidade é uma característica peculiar do segmento de fabricação de conservas de frutas que faz com que o postponement seja uma estratégia essencial à sobrevivência das empresas. Assim como a laranja, o tomate e a goiaba (também processado pela empresa T2) são produtos sazonais, sendo necessário o pré-processamento desses produtos no período de safra para abastecer a demanda na entressafra.

Apesar de, entre os direcionadores, a sazonalidade ser considerada um dos principais, ela não é o único direcionador que leva à postergação nas empresas pesquisadas. Outros direcionadores, embora não destacados por todas as empresas, foram também identificados como principais direcionadores para a adoção do postponement. Em ordem decrescente do número de empresas que citaram esses fatores com contribuição muito alta, podem-se destacar os seguintes: custo de produção e formas de acondicionamento do produto; custo de transporte, custo de estoque e custo de armazenagem; concentração de clientes no exterior, adoção da estratégia pelos concorrentes e variação de peso do produto; incerteza da demanda, marcas e versões do produto, prazo de validade do produto. Alguns desses direcionadores necessitam de maiores explicações e serão discutidos a seguir.

O desenvolvimento de novas formas de armazenamento e acondicionamento dos produtos, como a adoção do transporte a granel (para o suco de laranja) e dos bags assépticos (para as empresas produtoras de derivados de tomate), permitiram garantir isolamento dos produtos, possibilitando assim sua conservação por maior período de tempo, melhores formas de armazenagem e transporte, bem como a conservação das suas características naturais de sabor. Esses direcionadores auxiliaram a aplicação dos postponement nas empresas. 
Tabela 2. Direcionadores da adoção do postponement nas empresas de sucos e nas empresas de derivados de tomate.

\begin{tabular}{|c|c|c|c|c|c|c|c|}
\hline \multirow{2}{*}{ Dimensão } & \multirow{2}{*}{ Fatores } & \multicolumn{6}{|c|}{ Empresas } \\
\hline & & S1 & $\mathrm{S} 2$ & S3 & T1 & $\mathrm{T} 2$ & T3 \\
\hline \multirow{7}{*}{ Mercado } & Incerteza da demanda & 3 & 2 & 3 & 2 & 3 & 4 \\
\hline & Variação da demanda & 2 & 2 & 2 & 2 & 3 & 2 \\
\hline & Demanda para customização & 3 & 3 & 3 & 3 & 2 & 3 \\
\hline & Segmento de mercado & 2 & 2 & 2 & 2 & 2 & 2 \\
\hline & Consumidores exigentes & 0 & 1 & 0 & 2 & 1 & 2 \\
\hline & Concentração de clientes (no exterior) & 4 & 4 & 4 & 0 & 0 & 0 \\
\hline & Adoção da estratégia pelos concorrentes & 3 & 4 & 4 & 0 & 0 & 4 \\
\hline \multirow{15}{*}{ Produto } & Tipo do produto (sazonal) & 4 & 4 & 4 & 4 & 4 & 4 \\
\hline & Preço & 2 & 2 & 2 & 2 & 2 & 2 \\
\hline & Marcas e versões do produto (diferentes) & 3 & 3 & 4 & 3 & 3 & 4 \\
\hline & Variação de tamanho produto/embalagem & 3 & 3 & 3 & 3 & 3 & 3 \\
\hline & Variação de peso do produto (aumento) & 4 & 4 & 4 & 3 & 3 & 3 \\
\hline & Formulação específica do produto & 3 & 3 & 3 & 2 & 2 & 1 \\
\hline & Periféricos específicos & 0 & 0 & 0 & 0 & 0 & 0 \\
\hline & Modularidade/comonalidade & 0 & 0 & 0 & 0 & 0 & 0 \\
\hline & Padronização & 3 & 3 & 2 & 3 & 3 & 2 \\
\hline & Modelos intercambiáveis & 0 & 0 & 0 & 0 & 0 & 0 \\
\hline & Sequenciamento de peças & 0 & 0 & 0 & 0 & 0 & 0 \\
\hline & Complexidade e customização final (média) & 2 & 2 & 2 & 3 & 2 & 3 \\
\hline & Ciclo de vida & 1 & 2 & 2 & 3 & 3 & 2 \\
\hline & Prazo de validade & 2 & 3 & 3 & 3 & 3 & 4 \\
\hline & Estágio do ciclo de vida (maturação) & 1 & 1 & 2 & 2 & 1 & 2 \\
\hline \multirow{10}{*}{ Processo } & Processo modular & 0 & 0 & 0 & 0 & 0 & 0 \\
\hline & Processos de manufatura flexíveis & 2 & 2 & 1 & 2 & 2 & 1 \\
\hline & Complexidade tecnológica do processo & 1 & 1 & 1 & 1 & 1 & 2 \\
\hline & Reengenharia do processo de negócio & 0 & 0 & 0 & 0 & 0 & 1 \\
\hline & Planejamento de capacidade & 1 & 2 & 3 & 2 & 2 & 2 \\
\hline & Economia de escala & 2 & 2 & 3 & 2 & 2 & 3 \\
\hline & Custo da produção & 4 & 4 & 3 & 4 & 4 & 4 \\
\hline & Lead time & 2 & 2 & 2 & 2 & 2 & 2 \\
\hline & Desacoplamento do processo & 2 & 2 & 3 & 2 & 2 & 2 \\
\hline & Tecnologia de processo & 0 & 0 & 0 & 2 & 3 & 2 \\
\hline \multirow{10}{*}{ Logística } & Custo de estoque & 3 & 4 & 4 & 4 & 3 & 4 \\
\hline & Custo de armazenagem & 3 & 4 & 4 & 4 & 3 & 4 \\
\hline & Custo de transporte/distribuição & 3 & 4 & 4 & 4 & 3 & 4 \\
\hline & Custo de vendas perdidas & 2 & 2 & 2 & 2 & 2 & 3 \\
\hline & Infraestrutura de transporte & 2 & 2 & 2 & 2 & 3 & 2 \\
\hline & Serviço ao cliente & 2 & 2 & 2 & 2 & 2 & 2 \\
\hline & Tempo de entrega (30 a 40 dias) & 3 & 3 & 3 & 2 & 2 & 3 \\
\hline & Frequência de entrega & 2 & 2 & 2 & 2 & 2 & 2 \\
\hline & Incerteza de tempo de ressuprimento & 1 & 1 & 1 & 1 & 2 & 1 \\
\hline & Formas de acondicionamento do produto & 4 & 4 & 4 & 3 & 4 & 4 \\
\hline \multirow{6}{*}{ Cadeia de suprimentos } & Colaboração interfuncional & 2 & 2 & 2 & 2 & 2 & 2 \\
\hline & Colaboração com clientes/fornecedores & 2 & 2 & 3 & 1 & 2 & 1 \\
\hline & Resposta rápida dos fornecedores & 0 & 0 & 0 & 0 & 0 & 0 \\
\hline & Políticas governamentais & 0 & 0 & 0 & 0 & 0 & 0 \\
\hline & Treinamento e gestão da mudança & 0 & 0 & 0 & 0 & 1 & 0 \\
\hline & Medição de desempenho & 1 & 1 & 1 & 1 & 1 & 1 \\
\hline \multirow{2}{*}{ Liderança } & Estratégia da organização & 2 & 3 & 2 & 2 & 3 & 2 \\
\hline & Comprometimento & 3 & 3 & 3 & 3 & 3 & 3 \\
\hline \multirow{2}{*}{ Tecnologia } & E-commerce & 0 & 1 & 1 & 0 & 1 & 0 \\
\hline & Infraestrutura de tecnologia da informação & 3 & 3 & 3 & 3 & 3 & 2 \\
\hline
\end{tabular}


Em relação aos direcionadores como custos de produção e logística (custo de transporte, custo de estoque e custo de armazenagem), aumento de peso do produto após seu processamento final, diferentes marcas e versões foram também apontados entre os principais facilitadores e impulsionadores do uso do postponement nas empresas investigadas. Com a postergação de certas atividades no processo produtivo do suco de laranja, por exemplo, as empresas conseguiram reduzir o volume do produto a ser transportado e custos de produção e logísticos como: custo de estoque, de armazenagem, de transporte, diminuição do tempo de entrega, entre outros (dimensão logística). Adicionalmente, o risco de obsolescência no armazenamento do produto final é bem maior que do que no armazenamento do produto no estado semiacabado. 0 mesmo pôde ser observado pelas empresas produtoras de derivados de tomate.

Os direcionadores concentração de clientes no exterior e a adoção da estratégia pelos concorrentes também foram citados, principalmente pelas empresas processadoras de suco de laranja como sendo fatores com contribuição muito alta. 0 primeiro fator foi citado pois, de acordo com as empresas investigadas, existe maior consciência e demanda naqueles países por bebida natural e saudável, além de maior escassez de frutas in natura. Assim, as empresas processadoras de suco de laranja aplicam o conceito de postponement devido à possibilidade de se concentrarem na sua atividade principal, que é a obtenção da laranja, seu processamento e transformação em suco concentrado e congelado, deixando a atividade de reprocessamento, manufatura final e distribuição, que não são sua atividade fim, para outras empresas presentes nos países para os quais o produto é exportado. Já o fator adoção da estratégia pelos concorrentes foi citado pelas empresas S2, S3 e T3, pois quando foram fundadas seus principais concorrentes já adotavam o postponement. Assim, elas necessitaram também adotar a estratégia para manterem-se no mercado de forma competitiva. Atualmente, os entrevistados consideram que a aplicação do postponement já está consolidada no mercado e que, para essas empresas e para as demais deste segmento, mais que um diferencial, ela é essencial para a sobrevivência.

Há ainda fatores que, embora destacados na literatura, não se aplicam às empresas alimentícias investigadas e não foram citados, tais como: legislação, periféricos específicos, modularidade, modelos intercambiáveis, sequenciamento de peças, entre outros.

Além dos direcionadores considerados principais facilitadores e motivadores do postponement e classificados como de contribuição muito alta para a tomada de decisão pelas empresas da indústria alimentícia, foram destacados na Tabela 2 outros, classificados pelas empresas como de contribuição alta, média e baixa e que também são relevantes e devem ser considerados por empresas do setor alimentício que desejam adotar essa estratégia.

\section{Discussão dos resultados}

Baseado na pesquisa de campo foi possível responder a questão investigada por esta pesquisa: identificar como e que tipos de postponement são aplicados nas empresas alimentícias. Os resultados obtidos permitiram verificar a prática dos tipos de postponement de forma e de tempo selecionados da literatura nas empresas alimentícias investigadas, sendo o postponement de forma verificado prioritariamente na atividade de manufatura e não nas atividades de rotulagem e embalagem, como destacado na literatura.

Em relação ao estágio da cadeia de suprimentos em que o postponement acontece, foi possível verificar que nas empresas do segmento investigado isso acontece na própria empresa fabril ou à jusante da cadeia de suprimentos.

Nesta pesquisa foram destacados e discutidos os principais fatores considerados direcionadores para a aplicação do postponement. Destes, direcionadores como concentração de clientes no exterior (no caso das empresas de suco de laranja), adoção da estratégia pelos concorrentes, tecnologia de processo e formas de acondicionamento do produto foram observados nas empresas de sucos e produtoras de derivados de tomate, o que ainda não havia sido destacado na literatura sobre o tema.

Por outro lado, alguns direcionadores como políticas governamentais; periféricos específicos; modularidade/comonalidade; processo modular; modelos intercambiáveis; e sequenciamento de peças foram observados na literatura e não destacados pelos entrevistados. Em relação às políticas governamentais, os entrevistados não verificam nenhuma relação entre esse fator e a aplicação do postponement na indústria alimentícia. Já em relação aos outros direcionadores apontados na literatura como produto e processo modular, modelos intercambiáveis e sequenciamento de peças acredita-se que esses não foram apontados pelos entrevistados pela própria definição dos termos, que têm sua origem na indústria intermitente, como automobilística e eletrônica, e não porque sejam inexistentes. Starr (1965) definiu inicialmente o conceito de modularidade como uma abordagem de desenvolvimento de produtos em que o mesmo deve ser formado por meio da montagem de um conjunto de partes padronizadas. Embora nas empresas alimentícias pesquisadas não aconteça a montagem ou 
intercambiabilidade de peças padronizadas, a idéia da modularidade é aplicada também nesse setor, uma vez que uma condição para aplicação do postponement nas empresas pesquisadas é a existência do produto pré-preparado, ou seja, a existência de bases que geralmente são padronizadas. A partir do momento em que a demanda é conhecida, essas bases são utilizadas para a fabricação do produto final, que é diferenciado, ou seja, ocorre o processo de mistura (blendagem) para o suco de laranja e o processo de elaboração de receita para os derivados do tomate e da goiaba. Em ambos os casos, além da mistura, ocorre a adição de novos componentes, como, por exemplo, a essência, no caso do suco de laranja, e os temperos, no caso dos derivados de tomate, sendo essa atividade considerada parte do processo de manufatura (denominado manufatura final) nas empresas alimentícias investigadas. Assim, é possível verificar que as possibilidades de postergação em empresas de processo contínuo e em empresas de processo intermitente podem ser diferenciadas pelo conceito de modularidade. Enquanto no processo intermitente a modularidade do está associada àsua flexibilidade para a diferenciação do produto, nas empresas de processo contínuo, como as alimentícias, a possibilidade de obter o produto base (pré-preparado) é uma das condições para a diferenciação do produto posteriormente, seja por meio de atividades como a blendagem (no caso do suco de laranja), seja pela formulação e preparação de receitas, como no caso dos derivados de tomate e de goiaba. Em síntese, pode-se destacar que a possibilidade de elaboração do produto base (pré-preparado) bem como a possibilidade de futura diferenciação do produto (embora não explicitadas diretamente pelos entrevistados) também podem ser consideradas importantes motivadoras para aplicação do postponement em empresas alimentícias.

Em relação às especificidades do setor alimentício destacadas na introdução deste trabalho e agrupadas nas dimensões mercado, produto e processo, cabe destacar algumas delas e sua relação com a estratégia de postponement. Em relação ao mercado, as especificidades incerteza da demanda, concorrência e exportação de produtos destacadas na literatura por Van Donk (2001), Van Hoek (1999), Twede, Clarke e Tai (2000) e ABIA (ASSOCIAÇÃO..., 2011) para o setor alimentício foram também destacadas pelos entrevistados como os principais direcionadores da aplicação do postponement na dimensão mercado. Particularmente em relação à última característica (exportação de produtos), que pode ser observada nas empresas processadoras de suco de laranja analisadas, verificou-se que no ambiente de negócios brasileiro há grande oferta e abundância da fruta (laranja). Isso faz com que os consumidores ainda optem pelo suco natural em detrimento do suco processado. Assim, a maior parcela de suco concentrado e congelado produzida no Brasil é exportada para onde há escassez da fruta, sendo necessária a postergação de atividades de configuração final desse produto para empresas engarrafadoras localizadas no exterior. Em relação às particularidades do setor relacionadas ao produto, foram destacadas a sazonalidade e a pericibilidade, que estão associadas aos direcionadores sazonalidade e prazo de validade, destacados na pesquisa de campo. A sazonalidade foi o principal direcionador da adoção do postponement, destacado por todas as empresas. Já o prazo de validade está diretamente relacionado à pericibilidade do produto, ou seja, conforme já destacado, o postponement possibilita aumentar o prazo de validade dos produtos através de formas adequadas de processamento e armazenamento, reduzindo assim a sua pericibilidade. Por fim, embora autores como Van Hoek (1999) e Twede, Clarke e Tai (2000) afirmem que a não modularidade do produto e do processo ofereçam um desafio especial para a aplicação do postponement em alguns segmentos da indústria de alimento, foi possível verificar que embora nos segmentos investigados por esta pesquisa não exista o termo modularidade tal como conhecido nas empresas intermitentes, processos similares acontecem com outros rótulos. Conforme já destacado no parágrafo anterior, a modularidade está relacionada com a blendagem (mistura) de diferentes tipos de suco nas empresas de suco de laranja e com a elaboração de receitas nas empresas produtoras de derivados de tomate.

Nas empresas processadoras de suco de laranja concentrado e congelado e empresas produtoras de derivados de tomate brasileiras, o postponement é visto como uma questão de sobrevivência, uma vez que grande parte dos concorrentes dessas empresas já adota ou está em processo de adoção do postponement. Além disso, a maioria das empresas investigadas adota o postponement desde sua fundação, uma vez que a sazonalidade (característica deste segmento) requer o uso da estratégia para que os produtos possam ser oferecidos não somente na época de safra, mas durante $o$ ano inteiro.

Por fim, é importante também ressaltar que embora as empresas analisadas representem as maiores do segmento no Brasil, os resultados obtidos não podem ser generalizados para todo o segmento analisado. Pesquisas adicionais nas pequenas e médias empresas do mesmo segmento se fazem necessárias.

\section{Considerações finais}

Este estudo buscou aprofundar a pesquisa sobre postponement, principalmente em empresas da 
indústria de alimentos, setor onde existem poucas pesquisas e publicações sobre o tema. 0 propósito deste trabalho foi investigar a implementação do postponement e identificar os direcionadores da adoção dessa estratégia em empresas alimentícias. Para cumprir esses objetivos e responder às questões de investigação dessa pesquisa foi feito um esforço de levantamento da literatura relacionada à estratégia de postponement publicada nos últimos 60 anos. Adicionalmente foi realizado o estudo multicaso em seis empresas da indústria alimentícia.

Além de maior discussão sobre o tema e a verificação de sua aplicação em empresas alimentícias, este trabalho trouxe duas contribuições principais. A primeira foi a apresentação de um quadro teórico que apresenta os principais direcionadores para a adoção do postponement, que pode e deve ser adequado aos diferentes setores da indústria. A segunda foi a adequação desse quadro (com base na pesquisa de campo) para empresas da indústria alimentícia, especificamente do segmento de fabricação de conservas de frutas, bem como a apresentação de informações que fornecem subsídios para adoção e consolidação do postponement nessas empresas.

Destaca-se que apesar de haver poucas publicações sobre o tema no setor alimentício, a aplicação do postponement é uma prática cada vez mais frequente em empresas desse setor, particularmente no segmento de fabricação de conservas de frutas. Adicionalmente verifica-se que o postponement pode oferecer mais do que condições operacionais para o atendimento das necessidades individuais dos consumidores com rapidez e baixo custo. A adoção dessa estratégia tem também transformado as relações entre as empresas. Sua implementação implica, muitas vezes, em delegar atividades de diferenciação e/ou movimentação para outros integrantes da cadeia de suprimentos, conforme verificado nas empresas processadoras de suco de laranja investigadas. Nesse contexto, a adoção do postponement pode estreitar a relação entre os integrantes da cadeia de suprimentos, uma vez que ela exige mais coordenação e maior colaboração entre todos.

\section{Referências}

ALDERSON, W. Marketing efficiency and the principle of postponement. Cost and Profit Outlook, n. 3, p. 15-18, 1950.

ASSOCIAÇÃO BRASILEIRA DAS INDÚSTRIAS DE ALIMENTAÇÃO - ABIA. O setor em números. Sala de imprensa. São Paulo: ABIA, 20011. Disponível em: <http://www.abia.org.br>. Acesso em: fev. 2012.

ASSUMPÇÃO, M. R.; RIBEIRO, J. F. Compounding: postponement at processed food. In: INTERNATIONAL CONFERENCE ON AGRI-FOOD CHAIN/NETWORKS
ECONOMICS AND MANAGEMENT, 3., 2001, Ribeirão Preto. Anais... Ribeirão Preto, 2001.1 CD-ROM.

BALlOU, R. H. Gerenciamento da cadeia de suprimentos: planejamento, organização e logística empresarial. Porto Alegre: Bookman, 2001.

BOWERSOX, D. J.; ClOSS, D J. Logistical Management: the integrated supply chain process. New York: McGrawHill, 1996.

BUCKLIN, L. P. Postponement, speculation and the structure of distribution channels. Journal of Marketing Research, v. 2, p. 26-31, 1965. http://dx.doi.org/10.2307/3149333

CARDOSO, P. A. O princípio da postergação: um estudo na cadeia de suprimentos das tintas para impressão. 2002. 158 f. Tese (Doutorado em Engenharia de Produção)-Pontíficia Universidade Catolica do Rio de Janeiro, Rio de Janeiro, 2002.

CHIOU, J. S.; WU, L.; HSU, J. C. The adoption of form postponement strategy in a global logistics system: the case of Taiwanese information technology industry. Journal of Business Logistics, v. 32, n. 1, p. 107124, 2002. http://dx.doi.org/10.1002/j.2158-1592.2002. tb00018.x

COMISSÃO NACIONAL DE CLASSIFICAÇÃO - CONCLA. Classificação Nacional de Atividades Econômicas - CNAE. Tabela de códigos e classificações. IBGE, 2008. Disponível em: <http://www.cnae.ibge.gov.br>. Acesso em: ago. 2008.

COUNCIL OF LOGISTICS MANAGEMENT. World class logistics: the challenge of managing continuous change. United State of America: Oak Book, 1995.

GARCIA-DASTUGUE, S.; LAMBERT, D. Interorganizational time-based postponement in the supply chain. Journal of Business Logistics, v. 28, n. 1, p. 57-76, 2007. http:// dx.doi.org/10.1002/j.2158-1592.2007.tb00232.x

JOHNSON, M. E.; ANDERSON, E. Postponement strategies for channel derivatives. International Journal of Logistics Management, v.11, n. 1, p. 19-35, 2000. http://dx.doi. org/10.1108/09574090010806047

MACHADO, A. G. C; MORAES, W. F. A. Estratégias de customização em massa implementadas por empresas brasileiras. Revista Produção, v. 18, n. 1, p. 170-183, 2008.

MATTHEWS, P.; SYED, N. The power of postponement. Supply Chain Management, abr. 2004. Disponível em: <http://www2.isye.gatech.edu/ pinar/teaching/ isye3104-fall2004/postponement.pdf>. Acesso em: fev.2006.

PAGH, J. D.; COOPER, M. C. Postponement and speculation strategies: how to choose the right strategy. Journal of Business Logistics, v. 19, n. 2, p. 13-32, 1998.

SAMPAIO, M. O poder estratégico do postponement. 2003. 198 f. Tese (Doutorado em Administração)-Fundação Getúlio Vargas, São Paulo, 2003.

STARR, M. K. Modular-production: a new concept. Harvard Business Review, v. 43, n. 6, p. 131-142, 1965.

TWEDE, D.; CLARKE, R. H.; TAl, J. A. Packaging postponement: a global packaging strategy. Packaging Technology and Science, v. 13, p. 105-115, 2000. http://dx.doi. org/10.1002/1099-1522(200005) 13:3\%3C105::AlDPTS503\%3E3.0.C0;2-9

VAN DONK, D. P. Make to stock or make to order: the decoupling point in the food processing industries. 
International Journal Production Economics, v. 69, p. 297-306, 2001. http://dx.doi.org/10.1016/S09255273(00)00035-9

VAN HOEK, R. I. Postponed manufacturing: a case study in the food supply chain. Supply Chain Management, v. 2, n. 2, p. 18-34, 1997.

VAN HOEK, R. I. Postponement and the reconfiguration challenge for food supply chains. Supply Chain Management, v. 4, n. 1, p. 18-34, 1999. http://dx.doi. org/10.1108/13598549910255068

VAN HOEK, R. I. The rediscovery of postponement a literature review and directions for research. Journal of Operations Management, v. 19, n. 2, p. 161-184, 2001. http:// dx.doi.org/10.1016/S0272-6963(00)00057-7

VAN HOEK, V.; COMANDEUR, H. R.; VOS, B. Reconfiguring logistics systems through postponement strategies. Journal of Business logistics, v. 19, n. 1, p. 33-54, 1998.

VAN HOEK, R. 1.; PEELEN, E.; COMANDEUR, H. R. Achieving mass customization through postponement: a study of international changes. Journal of Market Focused Management, v. 3, p. 353-368, 1999. http://dx.doi. org/10.1023/A:1009827812231

WALlER, M. A.; DABHOLKAR, P. A.; GENTRY, J. J. Postponement, product, customization, and marketoriented supply chain management. Journal of Business Logistics, v. 21, n. 2, p. 133-156, 2000.

WIKNER, J.; NAIM, M. M.; RUDBERG, M. Exploiting the Order Book for Mass Customized Manufacturing Control
Systems With Capacity Limitations. IEEE Transactions on Engineering Management, v. 54, n. 1, p. 145-155, 2007. http://dx.doi.org/10.1109/TEM.2006.889073

YANG, B.; BURNS, N. D.; BACKHOUSE, C. J. Postponement: review and an integrated framework. International Journal of Operations e Production Management, v. 24, n. 5, p. 268-487, 2004a. http://dx.doi. org/10.1108/01443570410532542

YANG, B.; BURNS, N. D.; BACKHOUSE, C. J. Management of uncertainty through postponement. International Journal of Production Research, v. 42, n. 6, p. 10491064, 2004b. http://dx.doi.org/10.1080/002075403100 01631601

YANG, B.; BURNS, N. D.; BACKHOUSE, C. J. An empirical investigation into the barriers to postponement. International Journal of Production Research, v. 43, n. 5 , p. 991-1005, 2005. http://dx.doi.org/10.1080/0020754 0412331316134

YANG, B.; YANG,Y.; WIJNGAARD, J. Postponement: an inter-organizational perspective. International Journal of Production Research, v. 45, n. 4, p. 971-988, 2007. http://dx.doi.org/10.1080/00207540600698886

YIN, R. K. Estudo de caso: planejamento e métodos. 2. ed. Porto Alegre: Bookman, 2001.

ZINN, W.; BOWERSOX, D. J. Planning physical distribution with the principle of postponement. Journal of Business Logistics, v. 9, n. 2, p. 117-136, 1988.

\title{
Drivers in the adoption of postponement strategy: a multicase study in the food industry
}

\begin{abstract}
The purpose of this article is to investigate the practice of the postponement strategy in Brazilian food industry companies and identify which drivers that promote/facilitate the adoption of postponement in these companies. Six case studies were realized in processing and canning fruits and/or vegetables companies, such as orange juice and tomatoes processing companies. In addition to further discuss on the issue and verification of its application in food companies, this works presents two main contributions. The first is to present a theoretical framework with the main drivers for postponement strategy adoption based on the literature. The second is confronts the drivers obtained from empirical research and theory, generating the theoretical framework with the main drivers, adapted to the food industry.
\end{abstract}

\section{Keywords}

Postponement. Drivers. Supply chain management. 